\title{
Effect of COVID-19 outbreak on anxiety among students of higher education; A review of literature
}

\author{
Tony Jehi ${ }^{1}$ (1) $\cdot$ Raihan Khan ${ }^{1} \cdot$ Hildemar Dos Santos $^{2} \cdot$ Nader Majzoub $^{3}$ \\ Accepted: 29 November 2021 \\ (c) The Author(s), under exclusive licence to Springer Science+Business Media, LLC, part of Springer Nature 2021
}

\begin{abstract}
Background and Purpose The Covid-19 pandemic has impacted the mental health of students and exacerbated the prevalence of anxiety among them. The purpose of the literature review was to consolidate evidence for the prevalence of anxiety among students of higher education during the COVID-19 pandemic and to underscore the effect of various pandemic-related factors on this anxiety.

Methods A comprehensive literature search was conducted utilizing various databases such as MEDLINE, PsycInfo Google Scholar, ScienceDirect, Scopus, and Embase to identify relevant studies. To be incorporated in this review, studies had to include students of higher education, measure the prevalence of anxiety, and assess anxiety during Covid-19 pandemic.

Results 37 studies met the inclusion criteria and assessed the prevalence of anxiety among students during confinement. Studies showed that more than one-third of the students suffered from anxiety during the early stages of the pandemic. Moreover, being a female, living in rural areas, facing financial hardship, working full-time, spending the quarantine in isolation, worrying about infection for themselves and others, having the uncertainty of the future, having reduced sleep quality, and transitioning to online learning, were factors associated with increased anxiety during the pandemic.

Conclusion Anxiety was shown to be highly prevalent among the student population during the Covid-19 pandemic. Higher education institutions and governments should take action to ensure the safety and the physical, social, and mental wellbeing of the students.
\end{abstract}

Keywords Anxiety · Confinement · Covid-19 $\cdot$ Higher Education $\cdot$ Students

\section{Introduction}

Coronavirus disease of 2019 (Covid-19), a new coronavirus, emerged in the Chinese City of Wuhan in December 2019, then rapidly spread weeks later to reach other countries of the world, thus becoming a global threat. As a result, in the second week of March 2020, the World Health Organization (2020) in a media briefing, declared Covid-19 to be a pandemic. As of June 2021, there were

Tony Jehi

jehitr@jmu.edu

1 Department of Health Sciences, James Madison University, 235 M.L.K. Jr. Way, Harrisonburg, VA 22801, USA

2 Center for Nutrition, Healthy Lifestyle and Disease Prevention, School of Public Health, Loma Linda University, Loma Linda, CA, USA

3 KC Pharmaceuticals Inc, Pomona, CA, USA over 181 million confirmed cases and around 3 million and nine hundred thousand deaths (World Health Organization, 2021). This led health authorities to adopt various measures to fight and control the further spread of the virus. Among these, a principle, efficient yet extreme measure was the complete lockdown (Kraemer et al., 2020). A large-scale quarantine was mandated by many governments worldwide starting from March of 2020; unnecessary travel was banned and citizens were required to confine themselves to their homes.

Research carried out on previous epidemics has indicated that lockdowns and quarantine measures, albeit necessary, could engender negative psychological impacts such as depression, stress, and anxiety (Brooks et al., 2020). This is consistent with the findings of studies targeting the Covid-19 pandemic conducted on Chinese and Western societies (Cao et al., 2020; Rudenstine et al., 2021; C. Wang, Zhao, et al., 2020; Wang, Chen, 
et al., 2020; Wang, Hegde, et al., 2020; Wang, Yang, et al., 2020). Students, in particular, were among the populations that were most impacted by these psychological issues. It has been shown that students were vulnerable to mental health problems even preceding the pandemic (American College Health Association, 2015) due to academic challenges and various personal and psychosocial stressors. These include leaving the family home, building new social relationships, managing their time and multitasking, adjusting to new social and geographical environments, and allocating economic resources (Beiter et al., 2015). The lockdown accentuated these challenges further and exacerbated the mental wellbeing of students due to factors such as fear of infection (Garvey et al., 2021; Li et al., 2020), financial hardship (Lee et al., 2021; Sahile et al., 2020), physical inactivity(Deng et al., 2020; Xiang et al., 2020), challenges of relocation (Cao et al., 2020; Demetriou et al., 2021), and reduced sleep quality (Hamaideh et al., 2021; Saraswathi et al., 2020). Additionally, Emergency Remote Teaching (ERT) has also been shown to aggravate the psychological health issues of students (Cleofas \& Rocha, 2021; Hamaideh et al., 2021; Khawar et al., 2021).

Higher education institutions have shifted from faceto-face or blended to fully remote instruction termed as ERT to ensure its students continue to obtain the required education while shielding them from the spread of the virus. ERT is described by Hodges et al. (2020) as a "temporary shift of instructional delivery to an alternate delivery mode due to crisis circumstances". Suspending standard class-based teaching and transitioning to fully online instruction dramatically altered students' lives (Sahu) and disrupted their psychosocial functioning (Elmer et al., 2020). The hardship that ERT might have imposed combined with the sudden change in their lifestyles, the attenuation of social interaction, the financial challenges, and the uncertainty of the future, could have intensified the level of anxiety among students (Pragholapati, 2020; Rajkumar, 2020; Rossi et al., 2020; Tandon, 2020; Xiong et al., 2020).

Not much recent work has been done to assess the overall findings of studies investigating the impact of Covid-19 on anxiety levels among students and its associated factors. Thus, we conducted a literature review to draw from secondary data and consolidate evidence for the prevalence of anxiety of students during the Covid19 pandemic. Moreover, we searched existing literature to underscore the effect of various factors that have been linked to the pandemic on this anxiety level. We hypothesize an increase in the prevalence of anxiety among college students from the period preceding the pandemic to the period when lockdown was first initiated. We also hypothesize the association between this anxiety and various psychosocial factors such as diminished social interactions, fear of infection, financial hardship, and transition to online learning.

\section{Methods}

\section{Search and Inclusion Criteria}

A comprehensive literature search was conducted utilizing various databases such as MEDLINE, PsycInfo Google Scholar, ScienceDirect, Scopus, and Embase to identify relevant studies. The reference lists of the identified investigations were also used to find articles. The search was restricted from March 2020, which marks the start of the lockdown, to June 2021. Phrases used to find the related articles included "Covid-19 and Anxiety", "Online Learning and Anxiety", "Covid-19, Anxiety, and Students", and "Covid-19, Anxiety, and Higher Education".

To be incorporated in this review, studies had to: (i) be published in peer-reviewed journals, (ii) be written in English, (iii) include students of higher education, (iv) measure the prevalence of anxiety, and (v) assess anxiety during Covid-19 pandemic. We excluded investigations that did not include students of higher education, did not assess the prevalence of anxiety during the pandemic, and were review studies.

\section{Screening and Data Extraction}

As displayed in the flowchart, the initial screening for articles assessing prevalence of anxiety among students identified 583 citations; from these citations, 396 duplicates were excluded. The remaining 187 abstracts were screened from which 133 were excluded for reasons such as conference abstracts, the lack of full-texts, etc. The 54 full-text articles that remained were screened for eligibility, out of which 17 were then excluded based on the inclusion and exclusion criteria.

The following data were extracted from the papers: author, year of publication, students' major/field of study, date of data collection, country, sample size, assessment tool for measuring anxiety, and key findings. The extracted results included the prevalence of anxiety among the students of higher education during the pandemic and factors that explained/were associated with this anxiety; these included but were not limited to gender, financial status, place of residence, social interaction, sleep quality, and online learning. 


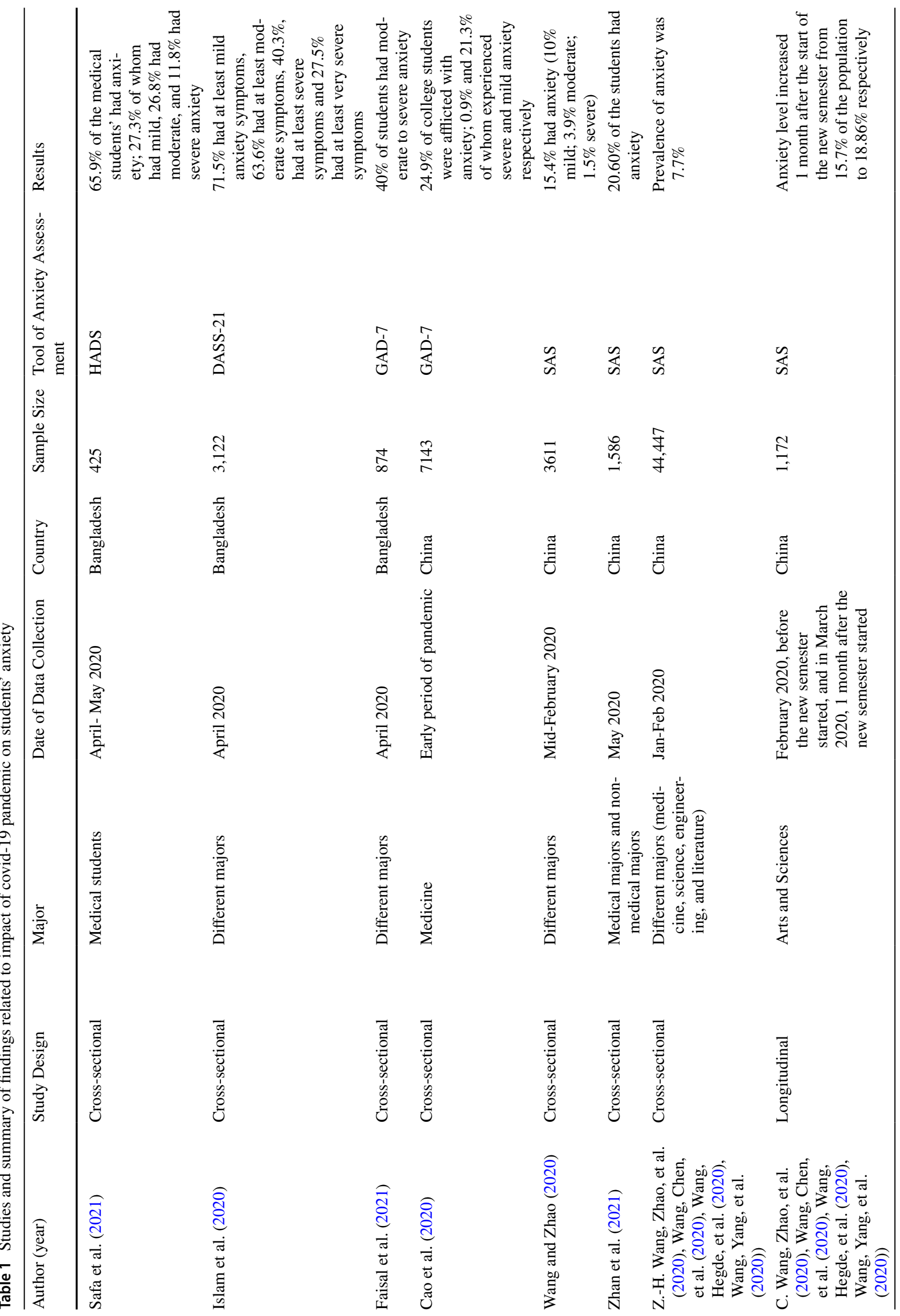




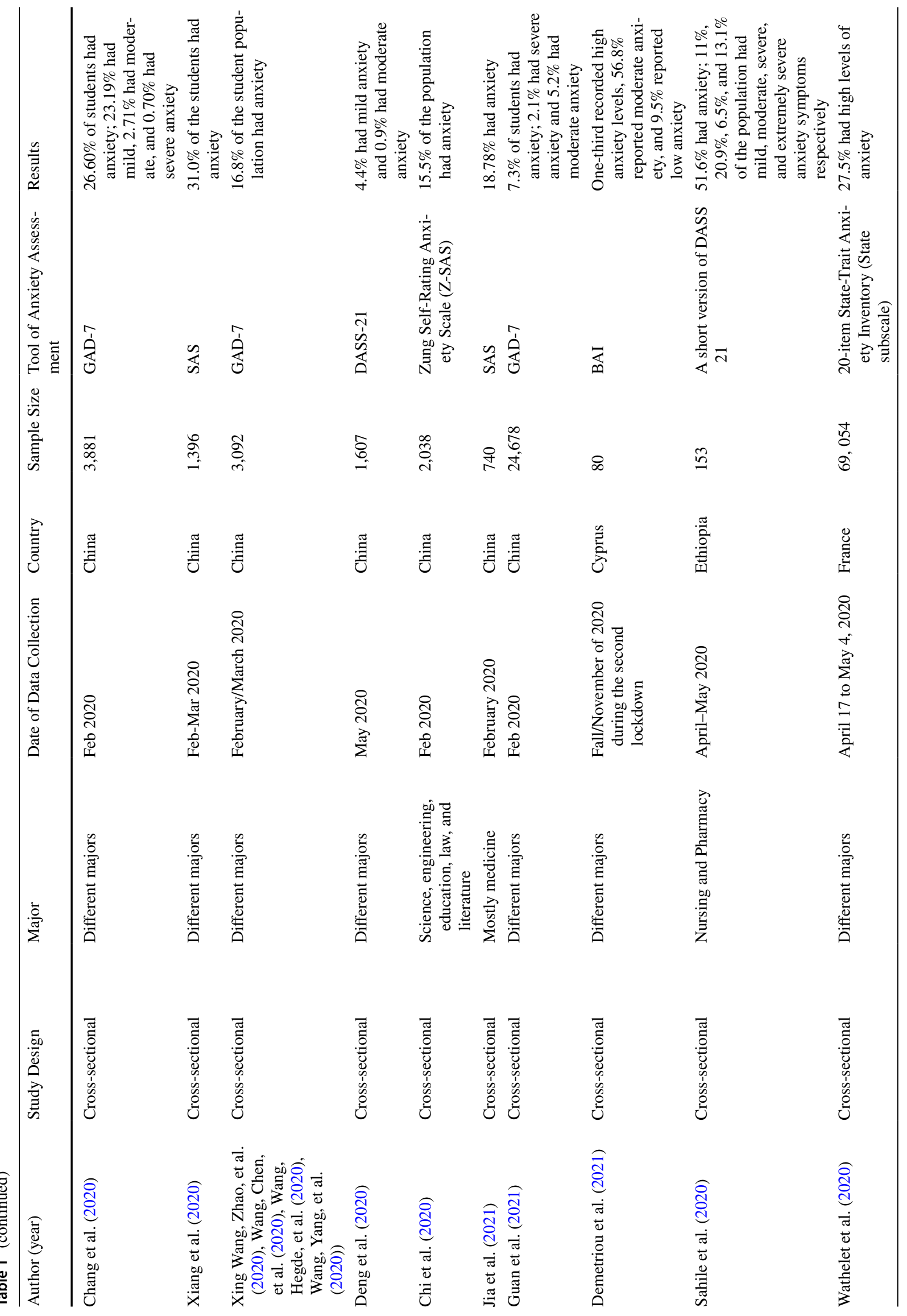




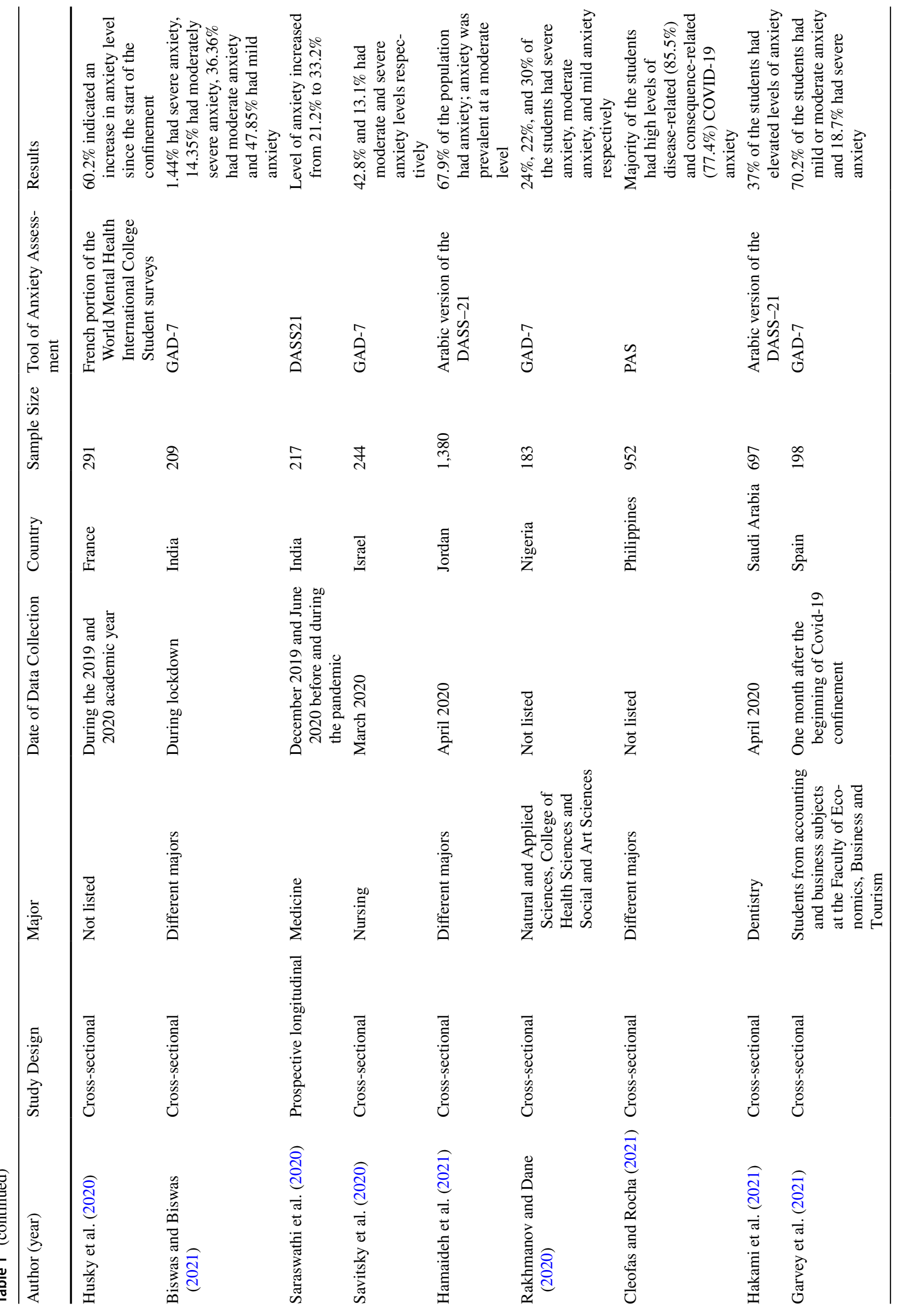




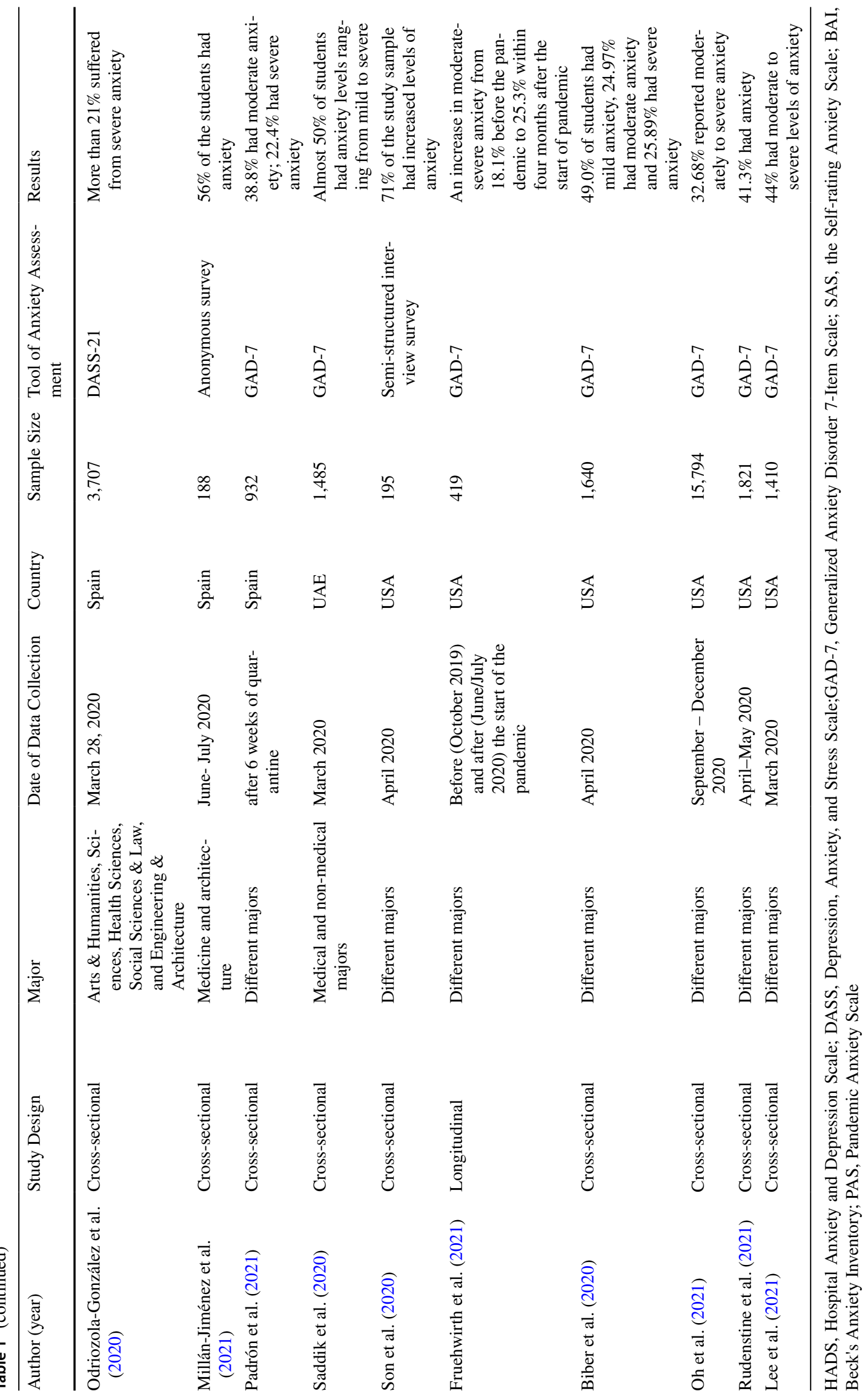




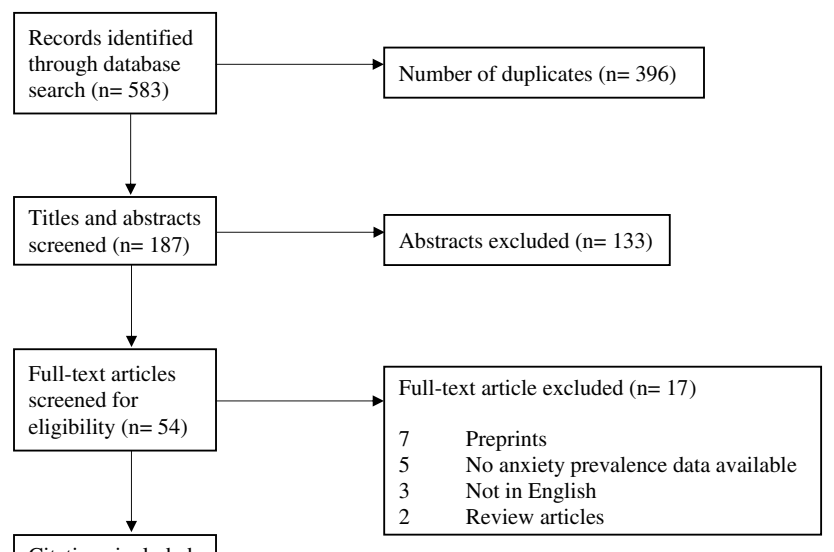

Citations included $(n=37)$

Fig. 1 Flowchart; screening process for the review of impact of Covid-19 on students' anxiety

\section{Results}

The results of the current literature review were based on the findings of existing published studies that investigated the prevalence of anxiety and its associated factors among students during the Covid-19 pandemic. Therefore, the findings were grouped into 2 themes; prevalence of anxiety and the factors associated with this anxiety during the Covid-19 confinement period.

\section{Anxiety Assessment and Prevalence}

We found that 37 studies have met the inclusion criteria and assessed the prevalence of anxiety among students during the pandemic. The majority of these studies were crosssectional, thus measuring its prevalence at one time point at an early stage of confinement; March to April of 2020. As presented in the table, findings were displayed based on the country where they were conducted. From the 37 investigations presented, 16 utilized the Generalized Anxiety Disorder 7-Item Scale (GAD-7) (Biber et al., 2020; Biswas \& Biswas, 2021; Cao et al., 2020; Chang et al., 2020; Faisal et al., 2021; Fruehwirth et al., 2021; Garvey et al., 2021; Guan et al., 2021; Lee et al., 2021; Oh et al., 2021; Padrón et al., 2021; Rakhmanov \& Dane, 2020; Rudenstine et al., 2021; Saddik et al., 2020; Savitsky et al., 2020; Xing Wang, Zhao, et al., 2020; Wang, Chen, et al., 2020; Wang, Hegde, et al., 2020; Wang, Yang, et al., 2020); 7 used a version of Depression, Anxiety, and Stress Scale (DASS) (Deng et al., 2020; Hakami et al., 2021; Hamaideh et al., 2021; Islam et al., 2020; Odriozola-González et al., 2020; Sahile et al., 2020; Saraswathi et al., 2020); 7 used the Self-rating Anxiety Scale (SAS) (Chi et al., 2020; Jia et al., 2021; Wang \&
Zhao, 2020; C. Wang, Zhao, et al., 2020; Wang, Chen, et al., 2020; Wang, Hegde, et al., 2020; Wang, Yang, et al., 2020; Z.-H. Wang, Zhao, et al., 2020; Wang, Chen, et al., 2020; Wang, Hegde, et al., 2020; Wang, Yang, et al., 2020; Xiang et al., 2020; Zhan et al., 2021); the remaining adopted a patient health questionnaire/survey to measure anxiety (Cleofas \& Rocha, 2021; Demetriou et al., 2021; Husky et al., 2020; Millán-Jiménez et al., 2021; Safa et al., 2021; Son et al., 2020; Wathelet et al., 2020). Students mainly were of varying majors including Medicine, Health Sciences, Social Sciences, Nursing, Dentistry, Business, Arts, Engineering, and Law (Table 1 and Fig. 1).

In Bangladesh, and as displayed in the table, results showed that during the early period of confinement, more than one-third of the student population had anxiety. Islam et al. (2020) indicated that over $71 \%$ of the students reported having at least mild anxiety symptoms on April 2020.

In China, findings were less consistent with anxiety prevalence ranging from as low as $4 \%$ (Deng et al., 2020) to as high as $31 \%$ (Xiang et al., 2020). The lack of consistency could possibly be related to the characteristics and lifestyles of the students; for instance, the low prevalence denoted by Deng et al. (2020) could be due to the high physical activity level of this study population which has been shown to be a protective factor against anxiety (Wathelet et al., 2020; Xiang et al., 2020). Evidence from longitudinal investigations that compared anxiety level before and during confinement, albeit scarce, confirmed a significant increase in its level. C. Wang, Zhao, et al. (2020), Wang, Chen, et al. (2020), Wang, Hegde, et al. (2020), Wang, Yang, et al. (2020)) for instance, indicated an increase in anxiety level from $15.7 \%$ to $18.86 \%$, between February 2020, before the new semester started, and March 2020, 1 month after the new semester started, respectively.

Studies on student populations in other countries such as Cyprus (Demetriou et al., 2021), Ethiopia (Sahile et al., 2020), France (Husky et al., 2020; Wathelet et al., 2020), India (Biswas \& Biswas, 2021; Saraswathi et al., 2020), Israel (Savitsky et al., 2020), Jordan (Hamaideh et al., 2021), Nigeria (Rakhmanov \& Dane, 2020), Philippines (Cleofas \& Rocha, 2021), Saudi Arabia (Hakami et al., 2021) and United Arab Emirates (UAE) (Saddik et al., 2020) showed varying levels of anxiety; however, there was agreement that no less than one-third of these populations had anxiety during the Covid-19 confinement. Overall, the more severe anxiety levels were less prevalent than mild and moderate anxiety (Biswas \& Biswas, 2021; Hamaideh et al., 2021; Rakhmanov \& Dane, 2020; Savitsky et al., 2020).

In Spain, similar to other populations, anxiety was prevalent among students during the Covid-19 lockdown (Garvey et al., 2021; Millán-Jiménez et al., 2021; OdriozolaGonzález et al., 2020; Padrón et al., 2021). Additionally, Millán-Jiménez et al. (2021) denoted that anxiety prevalence 
in Spain remained high even after the termination of the first Covid-19 lockdown on July 2020 with more than $55 \%$ of students reporting anxiety symptoms.

In USA, studies also have mostly confirmed the high prevalence of anxiety among students during the pandemic right after the lockdown was set forth in March/April 2020 (Biber et al., 2020; Lee et al., 2021; Rudenstine et al., 2021; Son et al., 2020). Although most of the investigations in the U.S. were cross-sectional (Biber et al., 2020; Lee et al., 2021; Oh et al., 2021; Rudenstine et al., 2021; Son et al., 2020), Fruehwirth et al. (2021) showed, in a longitudinal study, an increase in moderate-severe anxiety from $18.1 \%$ before the pandemic to $25.3 \%$ within four months after the start of the pandemic. Furthermore, Oh et al. (2021) indicated that even after the end of the lockdown, $32.68 \%$ of the students reported moderate to severe anxiety in USA on September 2020.

\section{Factors Associated with Anxiety}

In addition to the 37 articles displaying the prevalence of anxiety level among college students during the pandemic, the review encompassed other investigations that identified factors associated with this anxiety such as gender, academic level, fear of infection, financial hardship, residence, etc.

Gender The research findings are strongly consistent when it comes to the gender differences and indicate higher prevalence of anxiety for females compared to males; this has been observed in various populations (Biswas \& Biswas, 2021; Browning et al., 2021; Chang et al., 2020; Cleofas \& Rocha, 2021; Debowska et al., 2020; Fruehwirth et al., 2021; García-González et al., 2021; Garvey et al., 2021; Hakami et al., 2021; Hamaideh et al., 2021; Hoyt et al., 2021; Islam et al., 2020; Jia et al., 2021; Khoshaim et al., 2020; Kibbey et al., 2020; Lee et al., 2021; Li et al., 2021; Padrón et al., 2021; Rakhmanov \& Dane, 2020; Rudenstine et al., 2021; Saddik et al., 2020; Safa et al., 2021; Savitsky et al., 2020; Wang \& Zhao, 2020; C. Wang, Zhao, et al., 2020; Wang, Chen, et al., 2020; Wang, Hegde, et al., 2020; Wang, Yang, et al., 2020; Wathelet et al., 2020). In Spain for example, Garvey et al. (2021) conducted a study to assess the anxiety level of students at the University of Alcalá one month after the beginning of Covid-19 confinement and showed that the level of anxiety was higher among the female students compared to the male students. In China, similar findings were observed by Wang and Zhao (2020) who assessed the anxiety level of 3611 Chinese undergraduate university students.

It is not strongly established why females are more prone to anxiety compared to males; these gender differences could be due to various biological and psychosocial factors. For instance, females are more likely to be tasked with additional caretaking duties during the pandemic (Islam et al., 2020;
McLaren et al., 2020), suffer from financial hardship (Garvey et al., 2021; Wathelet et al., 2020), lose a job (Islam et al., 2020), have the responsibility of taking care of a child at home (Savitsky et al., 2020), and face social disintegration (Islam et al., 2020). Additionally, females have a higher perception of threat and a greater sensitivity to the loss of control compared to men which could render them more vulnerable to anxiety in situations of crisis and trauma (Olff et al., 2007).

Different Academic Levels There is an association between level of study and anxiety during the Covid-19 confinement period. Various investigations have compared the anxiety levels between students of different academic years, yet their findings lack consistency (Odriozola-González et al., 2020; Padrón et al., 2021; Xiaomei Wang, Zhao, et al., 2020; Wang, Chen, et al., 2020; Wang, Hegde, et al., 2020; Wang, Yang, et al., 2020; Z.-H. Wang, Zhao, et al., 2020; Wang, Chen, et al., 2020; Wang, Hegde, et al., 2020; Wang, Yang, et al., 2020). Odriozola-González et al. (2020) denoted a higher anxiety level among undergraduates compared to graduate students 2 weeks after the lockdown in the University of Valladolid, Spain. Xiaomei Wang, Zhao, et al. (2020), Wang, Chen, et al. (2020), Wang, Hegde, et al. (2020), Wang, Yang, et al. (2020)) confirmed this in a sample of students from Texas A\&M University. These differences could be explained by age and independence since undergraduate learning is less autonomous than graduate and since undergraduate students might have been more impacted by alterations in the teaching modality (Masten et al., 2006). Zhan et al. (2021), however, administered an online cross-sectional questionnaire survey to 1,586 Chinese college students in May 2020 and showed no significant difference in anxiety between graduates and non-graduates; this could be related to the small sample size of graduates in the study.

When it comes to comparing anxiety across different grades at the undergraduate level, results of various studies also lack consistency (Chang et al., 2020; Cleofas \& Rocha, 2021; Debowska et al., 2020; Hakami et al., 2021; Wang \& Zhao, 2020; C. Wang, Zhao, et al., 2020; Wang, Chen, et al., 2020; Wang, Hegde, et al., 2020; Wang, Yang, et al., 2020). In a large-scale, longitudinal, population-based survey conducted among college students in China, Li et al. (2021) found that senior students had a higher risk of developing anxiety during the pandemic compared to students of lower academic levels. Similar findings were also reported by Wang and Zhao (2020). These observed differences could be related to the senior students' uncertainty about the pandemic's impact on their graduation (Cornine, 2020) and future employment (Gao et al., 2020). The investigations by Cleofas and Rocha (2021) and Xiaomei Wang, Zhao, et al. (2020), Wang, Chen, et al. (2020), Wang, Hegde, et al. (2020), Wang, Yang, et al. (2020)), on the other hand, indicated that anxiety level was higher among freshmen and 
sophomore students compared to the senior students. Biswas and Biswas (2021) and Debowska et al. (2020) have also shown that anxiety was more prevalent among the younger students $(<20$ years of age) compared to the older students. This could possibly be explained by the association between older age and higher level of COVID-19 awareness (Chang et al., 2020). The higher the level of awareness of COVID-19 among the students, the more effectively they can practice prevention and control measures.

Rural vs. Urban Residence Due to the worldwide campus closures during the pandemic, students of higher education had the option of returning to their homes or remaining on campus in their student housing facilities. Research has indicated that the type of residence has an impact on anxiety (Cao et al., 2020; Chang et al., 2020; Lee et al., 2021; Ramos-Morcillo et al., 2020). Chang et al. (2020), Cao et al. (2020), and Lee et al. (2021) denoted that living in Urban areas is protective against anxiety while living in rural areas increases the risk of having anxiety symptoms. A qualitative study conducted by Ramos-Morcillo et al. (2020) on nursing students in Spain showed that the COVID confinement, particularly the transition to e-learning, was more worrying for those living in rural areas.

The imbalance of financial and educational resources between urban and rural areas could explain why cities are conducive to attenuating anxiety among the college student population. The urban economy is relatively affluent compared to the rural economy; cities, thus, offer better job opportunities and income stability (Shigemura et al., 2020). Cities also provide excellent educational resources while on the other hand, in certain deep rural areas, and due to the lack of electricity and the required technology, students might struggle with online learning (ATABHOTOR et al., 2020; Sarwar et al., 2020). Moreover, cities offer better sanitary conditions than towns and villages due to better access to cleaning and disinfecting products, which in-return mitigate worry of infection among students (Ayittey et al., 2020).

Financial Hardship The Covid-19 pandemic led to severe financial hardship due to the drastic rise in unemployment rates. According to the Congressional Research Service (2021), the unemployment rate in the United States reached 14.8\% in April 2020 which is considered the highest rate observed since the year 1948. Many lost their jobs, especially those employed in the leisure and hospitality sector as well as the education and services sector. Moreover, the economic crisis forced thousands of students to drop out of college and focus on finding means to generate income.

Research findings strongly agree that this financial hardship faced by students was associated with anxiety (Browning et al., 2021; Cao et al., 2020; Garvey et al., 2021; Islam et al., 2020; Lee et al., 2021; Oh et al., 2021; Rudenstine et al., 2021; Sahile et al., 2020; Savitsky et al., 2020; Wathelet et al., 2020). Wathelet et al. (2020) administered a survey, the 20-item State-Trait Anxiety Inventory (STAI), from April to May 2020 on 69,054 university students living in France during the COVID-19 quarantine and showed that loss/decrease of income was considered a risk factor for anxiety in this population. Cao et al. (2020) found that family income stability was a protective factor against anxiety among students in Changzhi medical college in China.

The financial hardship faced by students' families could generate the concern of not being able to pay tuition fees (Peng et al., 2012) and could lead them to seek full-time employment while attending school (Demetriou et al., 2021). Demetriou et al. (2021) indicated that working students experienced a higher anxiety level compared to those who were not employed. Moreover, the those who were full-time employees reported higher anxiety levels than those who worked parttime. This is explained by the added pressure of employment, the time shortage, and the challenges of adapting to the distance-learning methods while meeting the work tasks.

Living with Family Due to the drastic measures that higher education institutions implemented during the pandemic and the transition to online learning, many students chose to leave their place of residence on-campus and move back to their parents' home. Research strongly suggests that students who resided with their family during the pandemic had lower anxiety levels than those who spent the quarantine in solitude (Cao et al., 2020; Demetriou et al., 2021; Garvey et al., 2021; Husky et al., 2020; Wathelet et al., 2020). Cao et al. (2020) denoted that living with parents was considered a protective factor against anxiety among a group of medical students from Changzhi medical college in China.

Living with family during the lockdown might have attenuated the negative mental health impacts of quarantine as family offers the necessary emotional and financial support (Mariani et al., 2020). Additionally, students who relocated back to their parents home during the lockdown were more likely to have access to a yard, garden, or park (Husky et al., 2020). The home settings and the surrounding environment influence psychiatric symptoms (Millán-Jiménez et al., 2021). García-González et al. (2021) administered the Spielberger STAI during the first and fourth weeks of the confinement on nursing students at the University of Lorca (Murcia) and University of Almería (Spain) and indicated that those who spent confinement in a house with a garden had lower anxiety level than those living in a house without a garden.

Fear College students, even preceding the Covid-19 pandemic, experienced fear of examinations and academic failure, fear of not meeting the financial requirements of their degrees, and fear of the future's lack of employment opportunities (Bledsoe \& Baskin, 2014). The pandemic 
exacerbated these concerns and in return, increased their vulnerability to anxiety (Cao et al., 2020; Elmer et al., 2020; Garvey et al., 2021; Hasan \& Bao, 2020; Li et al., 2020; Oh et al., 2021; Saraswathi et al., 2020; Savitsky et al., 2020; Son et al., 2020; Wang \& Zhao, 2020). Savitsky et al. (2020) indicated that fear of infection and concern for the future were among the main factors positively associated with anxiety among a group of Israeli nursing students. Li et al. (2020) confirmed these findings in their longitudinal study which aimed to assess the mental health status changes of undergraduate students from Hebei Agricultural University in Baoding, China, before and after COVID-19 confinement. Results showed that fear of infection and inadequate supplies of alcohol hand rubs were significantly associated with an increase in anxiety level, 2 weeks after the start of confinement. Cao et al. (2020) suggested that having relatives or acquaintances infected with COVID-19, more than the fear of infection for oneself, was related to the prevalence of anxiety.

Research also suggests that students had intense academic fears due to the pandemic (Hamaideh et al., 2021; Hasan \& Bao, 2020; Son et al., 2020). Hasan and Bao (2020) showed that, in a group of Bangladeshi college students, transitioning to e-Learning and fear of academic year loss during the lockdown were related to higher levels of psychological anxiety.

Social Interactions The COVID-19 pandemic had an unfavorable effect on the social life of students as it diminished the strong social relations they established during the academic journey and confined them to their homes. Research suggests an association between the resulting weak social relations and the increased anxiety level (Fruehwirth et al., 2021; Hakami et al., 2021; Husky et al., 2020; Oliveira et al., 2021; Savitsky et al., 2020; Son et al., 2020; Wathelet et al., 2020). Switching to online learning during the pandemic negatively impacted students' mental health as it attenuated the teacher-student and student-student interactions (Fruehwirth et al., 2021; Oliveira et al., 2021).

Students who spent the confinement period in solitude were at higher risk of having mental problems compared to those who were not alone (Hakami et al., 2021; Hamaideh et al., 2021; Husky et al., 2020). Husky et al. (2020) showed that the anxiety level was higher among a group of French university students who did not relocate to another residence during the Covid-19 confinement period, and thus were more likely to be alone than those who did relocate. Studies have also underlined the importance of social support in combating mental issues (Cao et al., 2020; Demetriou et al., 2021). Cao et al. (2020), for instance, pointed out that students who were surrounded by friends and family reported less frequent terror symptoms during the lockdown than those who were living alone.
Physical Activity Research has shown that physical activity could protect against anxiety, especially during the pandemic (Deng et al., 2020; Osipov et al., 2021; Wathelet et al., 2020; Xiang et al., 2020). For instance, Xiang et al. (2020) indicated that a significant association exists between high level of physical activity, specifically, stretching and resistance training, and low anxiety level. Physical activity could attenuate anxiety symptoms by enhancing the immune system, improving the levels of neurotrophic factor (BDNF) and endogenous opioids (endorphins) and boosting self-esteem (Balchin et al., 2016). Due to the pandemic's lockdown, however, the physical activity level was reduced among the student population. Wathelet et al. (2020) demonstrated that being sedentary is a risk factor for anxiety in a group of students living in France during the COVID-19 quarantine.

Sleep Quality The reduction of sleep quality was directly associated with increased anxiety among the college student population during the Covid-19 pandemic (Garvey et al., 2021; Hamaideh et al., 2021; Islam et al., 2020; Saraswathi et al., 2020; Son et al., 2020; Xing Wang, Zhao, et al., 2020; Wang, Chen, et al., 2020; Wang, Hegde, et al., 2020; Wang, Yang, et al., 2020). Son et al. (2020) denoted that disruptions to sleeping patterns were related to the anxiety level. Islam et al. (2020) showed that having sleep dissatisfaction was one of the factors associated with higher anxiety levels among a group of Bangladeshi university students during the outbreak. These findings were confirmed by Xing Wang, Zhao, et al. (2020), Wang, Chen, et al. (2020), Wang, Hegde, et al. (2020), Wang, Yang, et al. (2020)) in their study which indicated that suffering from sleep issues was a risk factor for higher anxiety.

Online Learning Universities across the globe decided to implement emergency remote teaching during the confinement to ensure the safety of the faculty and students while offering the opportunity to continue their studies (Cleofas \& Rocha, 2021; Fawaz et al., 2021; Hamaideh et al., 2021; Khawar et al., 2021). This rapid transition was rife with challenges as it required technological adjustments, alterations in workplace culture, and changes in the students' learning modes and strategies.

College students were bombarded with many hurdles as they switched to online learning including the absence of formal/regular classes that offer a physical platform where they could attend and learn different subjects, the increase of academic workload (Fawaz et al., 2021), and the absence of a strong social and emotional connectivity (Demetriou et al., 2021). Such challenges may have eventually impacted their mental health (Cleofas \& Rocha, 2021; Demetriou et al., 2021; Fawaz et al., 2021; Hamaideh et al., 2021; Hasan \& Bao, 2020; Khawar et al., 2021; Oliveira et al., 2021; Saddik et al., 2020; Savitsky et al., 2020; Wang \& Zhao, 2020; C. 
Wang, Zhao, et al., 2020; Wang, Chen, et al., 2020; Wang, Hegde, et al., 2020; Wang, Yang, et al., 2020).

It is important though to first note the difference between online learning and ERT. Online learning usually requires months of preparation for courses designed to be taught in the online format; ERT, on the other hand, entails the rapid transformation of courses that might have been designed originally to be taught on-campus, to the online format due to an emergency such as the Covid-19 lockdown. For the purpose of the current systematic review though, the terms "online learning" and "ERT" were used interchangeably.

The transition from on-campus to e-learning occurred in a short period of time during the pandemic; teachers and scholars had to rapidly adapt to these changes which contributed to negative repercussions. Hasan and Bao (2020) denoted that e-Learning crack-up was associated with higher levels of psychological anxiety among Bangladeshi college students during the COVID-19 lockdown.

Many factors could affect the students' susceptibility to mental issues during the transition to e-learning including online class registration procedures, lack of knowledge and skills, one-way instructor support, satisfaction, fear of academic year loss, e-Learning content costs, increased workload, and technical issues. Cleofas and Rocha (2021) conducted a study to investigate the relationship between gadgets and Internet profiles and COVID-19 anxiety among Filipino college students. Results showed that those who did not own laptops or tablets and those who had limited or borrowed Internet connectivity suffered from higher levels of consequence-related COVID-19 anxiety. Lack of these tools rendered it challenging to complete the academic assigns and assessments, thus causing concern and worry of possible academic failure.

Additionally, Fawaz et al. (2021) indicated that transitioning to online learning had significantly increased workloads on students thus putting them at higher risk of anxiety and depression. Saddik et al. (2020) administered a surveyed on medical and non-medical university students in the UAE to assess anxiety at three time points: during hospital visits for medical students, before, and after the introduction of online learning. Anxiety level among medical students decreased after the introduction of online learning; as for the non-medical students, the anxiety level increased post initiation of online learning. The authors related this difference to various possible explanations including higher knowledge level of medical students when it comes to the use of online learning platforms and their reduced fear of infection after switching to e-learning.

Gaining the required skills and adapting to online learning is thus highly imperative. Demetriou et al. (2021) showed that students of a private university in Cyprus who were adapting to distance-learning methods reported lower anxiety levels than those still experiencing difficulties.
Furthermore, aside from knowledge and skill, Hamaideh et al. (2021) indicated that satisfaction with online learning was a protective factor against anxiety.

\section{Discussion}

More than one-third of college students suffered from anxiety during the early stages of the pandemic when the lockdown was first set forth on March/April of 2020 (Hamaideh et al., 2021; Islam et al., 2020; Safa et al., 2021; Savitsky et al., 2020). The prevalence of anxiety significantly increased from the period preceding the pandemic to the period when confinement was initiated (Saraswathi et al., 2020; C. Wang, Zhao, et al., 2020; Wang, Chen, et al., 2020; Wang, Hegde, et al., 2020; Wang, Yang, et al., 2020) and remained elevated even months later (Millán-Jiménez et al., 2021; Oh et al., 2021), especially in the Fall of 2020 during the second lockdown (Demetriou et al., 2021). Research on the risk factors associated with anxiety strongly suggest that being a female (García-González et al., 2021; Garvey et al., 2021; Padrón et al., 2021; Saddik et al., 2020; Savitsky et al., 2020; Wang \& Zhao, 2020; Wathelet et al., 2020), living in rural areas (Cao et al., 2020; Chang et al., 2020; Lee et al., 2021), facing financial hardship (Cao et al., 2020; Garvey et al., 2021; Savitsky et al., 2020; Wathelet et al., 2020), working full-time (Demetriou et al., 2021), spending the quarantine in solitude (Garvey et al., 2021; Husky et al., 2020; Wathelet et al., 2020), worrying about infection for themselves and others, having the uncertainty of the future (Bledsoe \& Baskin, 2014; Cao et al., 2020; Garvey et al., 2021; Li et al., 2020; Savitsky et al., 2020), having reduced sleep quality (Garvey et al., 2021; Islam et al., 2020; Son et al., 2020), and transitioning to online learning, were associated with increased anxiety among students during the pandemic. On the other hand, living in an urban area (Cao et al., 2020; Chang et al., 2020; Lee et al., 2021), spending quarantine in a house that has a yard or garden (García-González et al., 2021; Millán-Jiménez et al., 2021), receiving familial and social support (Garvey et al., 2021; Husky et al., 2020; Wathelet et al., 2020), building strong social relations (Fruehwirth et al., 2021; Hakami et al., 2021; Oliveira et al., 2021; Son et al., 2020), engaging in physical activity (Deng et al., 2020; Wathelet et al., 2020; Xiang et al., 2020), and adapting effectively to online learning, were protective against anxiety (Demetriou et al., 2021).

Addressing the psychological issues that have been exacerbated by the pandemic is imperative as previous research indicated their association with behavioral disorders such as substance use (Horigian et al., 2021) and suicide (De Berardis et al., 2018; Kanwar et al., 2013). Suicide, in particular, is a complex and multifaceted phenomenon that is 
challenging to comprehend since it is explained by a plethora of variables (Orsolini et al., 2020). There is existing evidence, however, indicating that having anxiety symptoms during the pandemic was a risk factor for suicidal ideation among university students (Zhou et al., 2021).

To our knowledge, this literature review is the first to take a comprehensive approach into identifying all of the citations, published globally, that address the Covid-19-related anxiety among students. It also drew from existing literature to present and consolidate the evidence for the factors rationalizing the high prevalence of anxiety among students during the pandemic. However, most of the displayed findings were generated from cross-sectional investigations which renders it challenging to assess causality and make robust conclusions (Biber et al., 2020; Biswas \& Biswas, 2021; Cao et al., 2020; Chang et al., 2020; Chi et al., 2020; Cleofas \& Rocha, 2021; Demetriou et al., 2021; Deng et al., 2020; Faisal et al., 2021; Garvey et al., 2021; Guan et al., 2021; Hakami et al., 2021; Hamaideh et al., 2021; Husky et al., 2020; Islam et al., 2020; Jia et al., 2021; Lee et al., 2021; Millán-Jiménez et al., 2021; Odriozola-González et al., 2020; Oh et al., 2021; Padrón et al., 2021; Rakhmanov \& Dane, 2020; Rudenstine et al., 2021; Saddik et al., 2020; Safa et al., 2021; Sahile et al., 2020; Savitsky et al., 2020; Son et al., 2020; Xing Wang, Zhao, et al., 2020; Wang, Chen, et al., 2020; Wang, Hegde, et al., 2020; Wang, Yang, et al., 2020; Z.-H. Wang, Zhao, et al., 2020; Wang, Chen, et al., 2020; Wang, Hegde, et al., 2020; Wang, Yang, et al., 2020; Wathelet et al., 2020; Xiang et al., 2020; Zhan et al., 2021). Anxiety levels were measured at one time point during the lockdown and were not evauated preceding the pandemic. Thus, there were no references to make a comparison and evaluate the changes in anxiety. Nevertheless, findings from various longitudinal studies that did assess the change in students' mental health before and during the pandemic suggest a significant increase in the anxiety level (Fruehwirth et al., 2021; García-González et al., 2021; Li et al., 2020; Saraswathi et al., 2020; C. Wang, Zhao, et al., 2020; Wang, Chen, et al., 2020; Wang, Hegde, et al., 2020; Wang, Yang, et al., 2020). Another limitation is the use of self-reported measures, in most of the studies, to assess and determine anxiety level of the students. Anxiety was thus not evaluated by a clinician following diagnostic evaluation but by administering various screening tools. The collected self-reported data could possibly be subjected to social desirability bias and memory recall bias (Biber et al., 2020; Faisal et al., 2021; Islam et al., 2020; Rudenstine et al., 2021; Safa et al., 2021; Savitsky et al., 2020). Additionally, we focused exclusively on anxiety in this review, and did not include other possible mental problems such as post-traumatic stress disorder and depression.

The findings of this literature review offer mental health professionals and public health policy makers valuable information on the impact of the Covid-19 lockdown on students' mental health which can assist them to be better prepared and take effective action in future outbreaks. We showed that even though confinement was necessary, it increased the prevalence of anxiety among students; we also underscored the various factors associated with this anxiety during lockdown, thus emphasizing the need to address each of them. Governments and higher education institutions should ensure that students feel safe and receive the required academic, social, mental, and financial support during confinement ( $\mathrm{Li}$ et al., 2020). They should strengthen the psychological monitoring and develop and implement a systematic mental health treatment plan (Faisal et al., 2021). Z.-H. Wang, Zhao, et al. (2020), Wang, Chen, et al. (2020), Wang, Hegde, et al. (2020), Wang, Yang, et al. (2020)) indicated that more than $40 \%$ of students reported the need to expand their psychological knowledge, to comprehend the common symptoms of anxiety, and to understand the means to alleviate the negative psychological effects associated with the pandemic. Extensive health education and health promotion activities should thus be carried out to empower students. Additionally, universities and colleges are required to encourage their students to engage regularly in physical activity as it combats anxiety and to regularly visit the on-campus psychological counseling centers. Mental health support must be provided through counseling centers free of charge during the pandemic and the gaps between the students and the counselors should be filled (Cohen et al., 2020). In case of future campus closures with subsequent pandemic waves, special attention must be paid to the psychological status of female students, those spending the confinement in solitude, and those facing financial hardship (Zhan et al., 2021). The faculty have an important role to play by offering their students the required accommodations to achieve academic success while minimizing stress and anxiety. They must create a smooth transition to online learning while offering a stable educational structure for students. This signifies perpetuating a stable educational framework, keeping the changes in teaching schedule to a minimum, announcing changes weeks ahead of time, utilizing asynchronous methods and video conferencing platforms to offer lectures, and connecting students with the colleges' technology office for guidance on advanced online learning management systems. They should embrace flexibility when it comes to assignment deadline policies and should give special consideration to students who are employed and/or taking care of a child (Savitsky et al., 2020). From a research standpoint, more longitudinal investigations should be conducted to assess the long-term effects of confinement on the psychological states of students (Odriozola-González et al., 2020). This would lead to stronger conclusions regarding the cause and effect relationships between the different factors presented in this review and the anxiety level among 
students of higher education. Furthermore, the research on other possible pandemic-related mental problems such as post-traumatic stress disorder and depression should be explored.

\section{Conclusion}

The Covid-19 outbreak has globally introduced numerous challenges for higher education institutions and exacerbated the students' mental health issues. Various factors associated with the pandemic such as financial hardship, weakened social interactions, reduced sleep quality, fear of infection, lack of physical activity, and loneliness have increased anxiety among college students. Thus, higher education institutions and governments should take action to ensure the safety and the physical, social, and mental wellbeing of the students. Public health and mental health professionals should design appropriate interventions at the group and individual levels to counteract anxiety and its consequences in this population. It is also imperative that future investigations further examine, longitudinally, the changes in anxiety levels preceding and throughout the pandemic.

Data Availability Not applicable.

Code Availability Not applicable.

\section{Declarations}

Ethical Statement Not applicable; no IRB was required for this literature review.

Conflict of Interest Disclosure None of the authors declared any conflict of interest.

Consent for Publication Not applicable.

\section{References}

American College Health Association. (2015). American college health association-national college health assessment II: Reference group executive summary spring 2015. Hanover, MD: American College Health Association, 132.

Atabhotor, I. S., Kofoworola, O. M., \& Bolatito, S. E. (2020). Challenges of e-learning amid COVID-19 lockdown in federal polytechnics, Nigeria.

Ayittey, F. K., Ayittey, M. K., Chiwero, N. B., Kamasah, J. S., \& Dzuvor, C. (2020). Economic impacts of Wuhan 2019-nCoV on China and the world. Journal of Medical Virology, 92(5), 473.

Balchin, R., Linde, J., Blackhurst, D., Rauch, H. L., \& Schönbächler, G. (2016). Sweating away depression? The impact of intensive exercise on depression. Journal of Affective Disorders, 200, 218-221.
Beiter, R., Nash, R., McCrady, M., Rhoades, D., Linscomb, M., Clarahan, M., \& Sammut, S. (2015). The prevalence and correlates of depression, anxiety, and stress in a sample of college students. Journal of Affective Disorders, 173, 90-96.

Biber, D. D., Melton, B., \& Czech, D. R. (2020). The impact of COVID-19 on college anxiety, optimism, gratitude, and course satisfaction. Journal of American College Health, 1-6.

Biswas, S., \& Biswas, A. (2021). Anxiety level among students of different college and universities in India during lock down in connection to the COVID-19 pandemic. Journal of Public Health, $1-7$.

Bledsoe, T. S., \& Baskin, J. J. (2014). Recognizing student fear: The elephant in the classroom. College Teaching, 62(1), 32-41.

Brooks, S. K., Webster, R. K., Smith, L. E., Woodland, L., Wessely, S., Greenberg, N., \& Rubin, G. J. (2020). The psychological impact of quarantine and how to reduce it: Rapid review of the evidence. The Lancet, 395(10227), 912-920.

Browning, M. H., Larson, L. R., Sharaievska, I., Rigolon, A., McAnirlin, O., Mullenbach, L., . . Reigner, N. (2021). Psychological impacts from COVID-19 among university students: Risk factors across seven states in the United States. PloS One, 16(1), e0245327.

Cao, W., Fang, Z., Hou, G., Han, M., Xu, X., Dong, J., \& Zheng, J. (2020). The psychological impact of the COVID-19 epidemic on college students in China. Psychiatry Research, 287, 112934.

Chang, J., Yuan, Y., \& Wang, D. (2020). Mental health status and its influencing factors among college students during the epidemic of COVID-19. Nan fang yi ke da xue xue bao= Journal of Southern Medical University, 40(2), 171-176.

Chi, X., Becker, B., Yu, Q., Willeit, P., Jiao, C., Huang, L., . . Lin, J. (2020). Prevalence and psychosocial correlates of mental health outcomes among Chinese college students during the coronavirus disease (COVID-19) pandemic. Frontiers in Psychiatry, 11, 803.

Cleofas, J. V., \& Rocha, I. C. N. (2021). Demographic, gadget and internet profiles as determinants of disease and consequence related COVID-19 anxiety among Filipino college students. Education and Information Technologies, 1-16.

Cohen, K. A., Graham, A. K., \& Lattie, E. G. (2020). Aligning students and counseling centers on student mental health needs and treatment resources. Journal of American College Health, 1-9.

Congressional Research Service. (2021). Unemployment Rates During the COVID-19 Pandemic. https://fas.org/sgp/crs/misc/R46554.pdf. Accessed June 2021.

Cornine, A. (2020). Reducing nursing student anxiety in the clinical setting: An integrative review. Nursing Education Perspectives, 41(4), 229-234.

De Berardis, D., Fornaro, M., Valchera, A., Cavuto, M., Perna, G., Di Nicola, M., . . Vellante, F. (2018). Eradicating suicide at its roots: preclinical bases and clinical evidence of the efficacy of ketamine in the treatment of suicidal behaviors. International journal of molecular sciences, 19(10), 2888.

Debowska, A., Horeczy, B., Boduszek, D., \& Dolinski, D. (2020), A repeated cross-sectional survey assessing university students' stress, depression, anxiety, and suicidality in the early stages of the COVID-19 pandemic in Poland. Psychological Medicine, 1-4.

Demetriou, L., Keramioti, L., \& Hadjicharalambous, D. (2021). Examining the relationship between distance learning processes and university students' anxiety in times of COVID-19. Demetriou, L., Hadjicharalambous, D., \& L. keramioti.(2021). Examining the relationship between distance learning processes and university students anxiety in times of covid. European Journal of Social Sciences Studies, 6(2), 123-141.

Deng, C.-H., Wang, J.-Q., Zhu, L.-M., Liu, H.-W., Guo, Y., Peng, X.-H., . . Xia, W. (2020). Association of web-based physical education with mental health of college students in Wuhan during the COVID-19 outbreak: cross-sectional survey study. Journal of Medical Internet Research, 22(10), e21301. 
Elmer, T., Mepham, K., \& Stadtfeld, C. (2020). Students under lockdown: Comparisons of students' social networks and mental health before and during the COVID-19 crisis in Switzerland. PloS One, 15(7), e0236337.

Faisal, R. A., Jobe, M. C., Ahmed, O., \& Sharker, T. (2021). Mental health status, anxiety, and depression levels of Bangladeshi university students during the COVID-19 pandemic. International journal of mental health and addiction, 1-16.

Fawaz, M., Al Nakhal, M., \& Itani, M. (2021). COVID-19 quarantine stressors and management among Lebanese students: a qualitative study. Current Psychology, 1-8.

Fruehwirth, J. C., Biswas, S., \& Perreira, K. M. (2021). The Covid-19 pandemic and mental health of first-year college students: Examining the effect of Covid-19 stressors using longitudinal data. PloS One, 16(3), e0247999.

Gao, J., Zheng, P., Jia, Y., Chen, H., Mao, Y., Chen, S., . . Dai, J. (2020). Mental health problems and social media exposure during COVID-19 outbreak. PloS One, 15(4), e0231924.

García-González, J., Ruqiong, W., Alarcon-Rodriguez, R., RequenaMullor, M., Ding, C., \& Ventura-Miranda, M. I. (2021). Analysis of Anxiety Levels of Nursing Students Because of e-Learning during the COVID-19 Pandemic. Healthcare,

Garvey, A. M., García, I. J., Otal Franco, S. H., \& Fernández, C. M. (2021). The psychological impact of strict and prolonged confinement on business students during the COVID-19 pandemic at a Spanish University. International Journal of Environmental Research and Public Health, 18(4), 1710.

Guan, J., Wu, C., Wei, D., Xu, Q., Wang, J., Lin, H., . . Mao, Z. (2021). Prevalence and factors for anxiety during the COVID-19 pandemic among college students in China. International Journal of Environmental Research and Public Health, 18(9), 4974.

Hakami, Z., Khanagar, S. B., Vishwanathaiah, S., Hakami, A., Bokhari, A. M., Jabali, A. H., . . . Aldrees, A. M. (2021). Psychological impact of the coronavirus disease 2019 (COVID-19) pandemic on dental students: a nationwide study. Journal of Dental Education, 85(4), 494-503.

Hamaideh, S. H., Al-Modallal, H., Tanash, M. a., \& Hamdan-Mansour, A. (2021). Depression, anxiety and stress among undergraduate students during COVID-19 outbreak and "home-quarantine". Nursing Open.

Hasan, N., \& Bao, Y. (2020). Impact of "e-Learning crack-up" perception on psychological distress among college students during COVID-19 pandemic: A mediating role of "fear of academic year loss". Children and Youth Services Review, 118, 105355.

Hodges, C., Moore, S., Lockee, B., Trust, T., \& Bond, A. (2020). The difference between emergency remote teaching and online learning. Educause Review, 27(1), 1-9.

Horigian, V. E., Schmidt, R. D., \& Feaster, D. J. (2021). Loneliness, mental health, and substance use among US young adults during COVID-19. Journal of Psychoactive Drugs, 53(1), 1-9.

Hoyt, L. T., Cohen, A. K., Dull, B., Castro, E. M., \& Yazdani, N. (2021). "Constant stress has become the new normal": Stress and anxiety inequalities among US College students in the time of covid-19. Journal of Adolescent Health, 68(2), 270-276.

Husky, M. M., Kovess-Masfety, V., \& Swendsen, J. D. (2020). Stress and anxiety among university students in France during Covid-19 mandatory confinement. Comprehensive Psychiatry, 102, 152191.

Islam, M. S., Sujan, M. S. H., Tasnim, R., Sikder, M. T., Potenza, M. N., \& Van Os, J. (2020). Psychological responses during the COVID-19 outbreak among university students in Bangladesh. PloS One, 15(12), e0245083.

Jia, Y., Qi, Y., Bai, L., Han, Y., Xie, Z., \& Ge, J. (2021). Knowledgeattitude-practice and psychological status of college students during the early stage of COVID-19 outbreak in China: a crosssectional study. BMJ open, 11(2), e045034.

Kanwar, A., Malik, S., Prokop, L. J., Sim, L. A., Feldstein, D., Wang, Z., \& Murad, M. H. (2013). The association between anxiety disorders and suicidal behaviors: A systematic review and metaanalysis. Depression and Anxiety, 30(10), 917-929.

Khawar, M. B., Abbasi, M. H., Hussain, S., Riaz, M., Rafiq, M., Mehmood, R., . . . Jabeen, F. (2021). Psychological impacts of COVID-19 and satisfaction from online classes: disturbance in daily routine and prevalence of depression, stress, and anxiety among students of Pakistan. Heliyon, 7(5), e07030.

Khoshaim, H. B., Al-Sukayt, A., Chinna, K., Nurunnabi, M., Sundarasen, S., Kamaludin, K., . . Hossain, S. F. A. (2020). Anxiety level of University students during COVID-19 in Saudi Arabia. Frontiers in Psychiatry, 11, 1397.

Kibbey, M. M., Fedorenko, E. J., \& Farris, S. G. (2020). Anxiety, depression, and health anxiety in undergraduate students living in initial US outbreak "hotspot" during COVID-19 pandemic. Cognitive Behaviour Therapy, 1-13.

Kraemer, M. U., Yang, C.-H., Gutierrez, B., Wu, C.-H., Klein, B., Pigott, D. M., . . Hanage, W. P. (2020). The effect of human mobility and control measures on the COVID-19 epidemic in China. Science, 368(6490), 493-497.

Lee, J., Jeong, H. J., \& Kim, S. (2021). Stress, anxiety, and depression among undergraduate students during the COVID-19 pandemic and their use of mental health services. Innovative higher education, 1-20.

Li, H. Y., Cao, H., Leung, D. Y., \& Mak, Y. W. (2020). The psychological impacts of a COVID-19 outbreak on college students in China: A longitudinal study. International Journal of Environmental Research and Public Health, 17(11), 3933.

Li, Y., Zhao, J., Ma, Z., McReynolds, L. S., Lin, D., Chen, Z., . . . Zhang, J. (2021). Mental health among college students during the COVID-19 pandemic in China: A 2-wave longitudinal survey. Journal of Affective Disorders, 281, 597-604.

Mariani, R., Renzi, A., Di Trani, M., Trabucchi, G., Danskin, K., \& Tambelli, R. (2020). The impact of coping strategies and perceived family support on depressive and anxious symptomatology during the coronavirus pandemic (COVID-19) lockdown. Frontiers in Psychiatry, 11, 1195.

Masten, A. S., Obradović, J., \& Burt, K. B. (2006). Resilience in emerging adulthood: Developmental perspectives on continuity and transformation.

McLaren, H. J., Wong, K. R., Nguyen, K. N., \& Mahamadachchi, K. N. D. (2020). Covid-19 and women's triple burden: Vignettes from Sri Lanka, Malaysia, Vietnam and Australia. Social Sciences, 9(5), 87.

Millán-Jiménez, A., Herrera-Limones, R., López-Escamilla, Á., LópezRubio, E., \& Torres-García, M. (2021). Confinement, comfort and health: Analysis of the real influence of lockdown on university students during the COVID-19 pandemic. International Journal of Environmental Research and Public Health, 18(11), 5572.

Odriozola-González, P., Planchuelo-Gómez, Á., Irurtia, M. J., \& de Luis-García, R. (2020). Psychological effects of the COVID-19 outbreak and lockdown among students and workers of a Spanish university. Psychiatry Research, 290, 113108.

Oh, H., Marinovich, C., Rajkumar, R., Besecker, M., Zhou, S., Jacob, L., . . Smith, L. (2021). COVID-19 dimensions are related to depression and anxiety among US college students: Findings from the Healthy Minds Survey 2020. Journal of Affective Disorders.

Olff, M., Langeland, W., Draijer, N., \& Gersons, B. P. (2007). Gender differences in posttraumatic stress disorder. Psychological Bulletin, 133(2), 183.

Oliveira, L., Mesquita, A., Sequeira, A., \& Oliveira, A. (2021). Emergency remote learning during COVID-19: Socio-educational impacts on Portuguese students. Educating Engineers for Future Industrial Revolutions, 1328, 303.

Orsolini, L., Latini, R., Pompili, M., Serafini, G., Volpe, U., Vellante, F., . . Fraticelli, S. (2020). Understanding the complex of suicide in depression: from research to clinics. Psychiatry Investigation, 17(3), 207 
Osipov, A. Y., Ratmanskaya, T. I., Zemba, E. A., Potop, V., Kudryavtsev, M. D., \& Nagovitsyn, R. S. (2021). The impact of the universities closure on physical activity and academic performance in physical education in university students during the COVID-19 pandemic. Physical Education of Students, 25(1), 20-27.

Padrón, I., Fraga, I., Vieitez, L., Montes, C., \& Romero, E. (2021). A study on the psychological wound of COVID-19 in university students. Frontiers in Psychology, 12, 9.

Peng, L., Zhang, J., Li, M., Li, P., Zhang, Y., Zuo, X., . . Xu, Y. (2012). Negative life events and mental health of Chinese medical students: the effect of resilience, personality and social support. Psychiatry Research, 196(1), 138-141.

Pragholapati, A. (2020). COVID-19 impact on students.

Rajkumar, R. P. (2020). COVID-19 and mental health: A review of the existing literature. Asian Journal of Psychiatry, 52, 102066.

Rakhmanov, O., \& Dane, S. (2020). Knowledge and anxiety levels of African university students against COVID-19 during the pandemic outbreak by an online survey. Journal of Research in Medical and Dental Science, 8(3), 53-56.

Ramos-Morcillo, A. J., Leal-Costa, C., Moral-García, J. E., \& RuzafaMartínez, M. (2020). Experiences of nursing students during the abrupt change from face-to-face to e-learning education during the first month of confinement due to COVID-19 in Spain. International Journal of Environmental Research and Public Health, 17(15), 5519.

Rossi, R., Socci, V., Talevi, D., Mensi, S., Niolu, C., Pacitti, F., . . Di Lorenzo, G. (2020). COVID-19 pandemic and lockdown measures impact on mental health among the general population in Italy. Frontiers in Psychiatry, 11, 790.

Rudenstine, S., McNeal, K., Schulder, T., Ettman, C. K., Hernandez, M., Gvozdieva, K., \& Galea, S. (2021). Depression and anxiety during the covid-19 pandemic in an urban, low-income public university sample. Journal of Traumatic Stress, 34(1), 12-22.

Saddik, B., Hussein, A., Sharif-Askari, F. S., Kheder, W., Temsah, M.-H., Koutaich, R. A., . . Hamid, Q. (2020). Increased levels of anxiety among medical and non-medical university students during the COVID-19 pandemic in the United Arab Emirates. Risk management and healthcare policy, 13, 2395.

Safa, F., Anjum, A., Hossain, S., Trisa, T. I., Alam, S. F., Rafi, M. A., . . Ahmad, W. U. (2021). Immediate psychological responses during the initial period of the COVID-19 pandemic among Bangladeshi medical students. Children and Youth Services Review, 122,105912 .

Sahile, A. T., Ababu, M., Alemayehu, S., Abebe, H., Endazenew, G., Wubshet, M., . . . Tadesse, T. (2020). Prevalence and severity of depression, anxiety, and stress during pandemic of COVID-19 among college students in Addis Ababa, Ethiopia, 2020: a cross sectional survey. Int J Clin Exp Med Sci, 6(6), 126.

Sahu, P. Closure of universities due to coronavirus disease 2019 (COVID-19): Impact on education and mental health of students and academic staff. Cureus. 2020; 12 (4): e7541. Eng.) https://doi. org/10.7759/cureus, 7541 .

Saraswathi, I., Saikarthik, J., Kumar, K. S., Srinivasan, K. M., Ardhanaari, M., \& Gunapriya, R. (2020). Impact of COVID-19 outbreak on the mental health status of undergraduate medical students in a COVID-19 treating medical college: a prospective longitudinal study. PeerJ, 8, e10164.

Sarwar, H., Akhtar, H., Naeem, M. M., Khan, J. A., Waraich, K., Shabbir, S., . . . Khurshid, Z. (2020). Self-reported effectiveness of e-Learning classes during COVID-19 pandemic: A nation-wide survey of Pakistani undergraduate dentistry students. European journal of dentistry.

Savitsky, B., Findling, Y., Ereli, A., \& Hendel, T. (2020). Anxiety and coping strategies among nursing students during the covid-19 pandemic. Nurse Education in Practice, 46, 102809.
Shigemura, J., Ursano, R. J., Morganstein, J. C., Kurosawa, M., \& Benedek, D. M. (2020). Public responses to the novel 2019 coronavirus (2019-nCoV) in Japan: Mental health consequences and target populations. Psychiatry and Clinical Neurosciences, 74(4), 281.

Son, C., Hegde, S., Smith, A., Wang, X., \& Sasangohar, F. (2020). Effects of COVID-19 on college students' mental health in the United States: Interview survey study. Journal of Medical Internet Research, 22(9), e21279.

Tandon, R. (2020). COVID-19 and mental health: preserving humanity, maintaining sanity, and promoting health. Asian Journal of Psychiatry, 51, 102256.

Wang, C., \& Zhao, H. (2020). The impact of COVID-19 on anxiety in Chinese university students. Frontiers in Psychology, 11, 1168.

Wang, C., Zhao, H., \& Zhang, H. (2020). Chinese college students have higher anxiety in new semester of online learning during COVID-19: A machine learning approach. Frontiers in Psychology, 11, 3465 .

Wang, X., Chen, H., Liu, L., Liu, Y., Zhang, N., Sun, Z., . . Li, M. (2020). Anxiety and sleep problems of college students during the outbreak of COVID-19. Frontiers in Psychiatry, 11.

Wang, X., Hegde, S., Son, C., Keller, B., Smith, A., \& Sasangohar, F. (2020). Investigating mental health of US college students during the COVID-19 pandemic: cross-sectional survey study. Journal of Medical Internet Research, 22(9), e22817.

Wang, Z.-H., Yang, H.-L., Yang, Y.-Q., Liu, D., Li, Z.-H., Zhang, X.-R., . . . Song, W.-Q. (2020). Prevalence of anxiety and depression symptom, and the demands for psychological knowledge and interventions in college students during COVID-19 epidemic: A large cross-sectional study. Journal of Affective Disorders, 275, 188-193.

Wathelet, M., Duhem, S., Vaiva, G., Baubet, T., Habran, E., Veerapa, E., . . . Grandgenèvre, P. (2020). Factors associated with mental health disorders among university students in France confined during the COVID-19 pandemic. JAMA network open, 3(10), e2025591-e2025591.

World Health Organization. (2020). WHO Director-General's opening remarks at the media briefing on COVID-19 - 11 March 2020. https://www.who.int/director-general/speeches/detail/ who-director-general-s-opening-remarks-at-the-media-briefingon-covid-19---11-march-2020. Accessed June 2021.

World Health Organization. (2021). WHO Coronavirus (COVID-19) Dashboard. https://covid19.who.int/. Accessed June 2021.

Xiang, M.-Q., Tan, X.-M., Sun, J., Yang, H.-Y., Zhao, X.-P., Liu, L., . . . Hu, M. (2020). Relationship of physical activity with anxiety and depression symptoms in Chinese college students during the COVID-19 outbreak. Frontiers in Psychology, 11, 2860.

Xiong, J., Lipsitz, O., Nasri, F., Lui, L. M., Gill, H., Phan, L., . . . Majeed, A. (2020). Impact of COVID-19 pandemic on mental health in the general population: A systematic review. Journal of Affective Disorders.

Zhan, H., Zheng, C., Zhang, X., Yang, M., Zhang, L., \& Jia, X. (2021). Chinese college students' stress and anxiety levels under COVID19. Frontiers in Psychiatry, 12.

Zhou, S.-J., Wang, L.-L., Qi, M., Yang, X.-J., Gao, L., Zhang, S.-Y., , . Chen, J.-X. (2021). Depression, Anxiety, and Suicidal Ideation in Chinese University Students During the COVID-19 Pandemic. Frontiers in Psychology, 3389.

Publisher's note Springer Nature remains neutral with regard to jurisdictional claims in published maps and institutional affiliations. 